\title{
ESTIMACIÓN DE PRECIOS DE EQUILIBRIO EN UN MODELO GENERALIZADO DE INSUMO-PRODUCTO *
}

\author{
JAIME SERRA \\ El Colegio de México
}

\section{INTRODUCCIÓN}

Los MODELos de insumo-producto por lo común se representan mediante matrices que no contienen elementos que corresponden a producción conjunta. ${ }^{1}$ En particular, el conocido modelo de Leontief se representa por tal tipo de matriz, la cual, en adición, tiene la propiedad de que las actividades de producción sólo utilizan un factor escaso. Dadas estas características, existen métodos relativamente simples que permiten la estimación de precios de equilibrio. El cálculo de tales precios requiere la inversión de la matriz de insumo-producto, procedimiento único para la determinación de dichos precios.

Considerar sistemas con más de un factor escaso conduce a situaciones donde esta "afortunada propiedad" - la cual se explica más adelantese pierde. Los precios relativos de los bienes o productos cambian con los diferentes precios de los factores cuando existe más de un factor escaso: la estimación de los precios de equilibrio requiere considerar el factor mercado.

En este trabajo se presenta un algoritmo para estimar los precios de equilibrio (tanto para bienes como para factores productivos) en un modelo que no contiene producción conjunta pero en el que se utiliza más de un factor escaso. En este algoritmo se combinan las propiedades de la programación lineal con las del algoritmo de Scarf (1973).

En la siguiente sección (II); en la que se describe el algoritmo, se muestra la imposibilidad de estimar los precios de equilibrio sólo con la matriz de coeficientes técnicos (matriz A); en la sección III se describe el algoritmo utilizado y se señalan algunas consideraciones sobre sus propiedades; en la sección IV se presenta un ejemplo que muestra las principales etapas del algoritmo así como su grado de exactitud y; por último,

* Este trabajo fue escrito para el curso de Economía Matemática con la su. pervisión del profesor H. Scarf, Universidad Yale, 1977.

I Cada industria o sector produce un solo artículo. 
se presenta un resumen de los principales resultados derivados de la utilización del algoritmo mencionado y de sus posibles implicaciones.

\section{Cambios en los precios Relativos}

Una economía que es representada por una matriz de producción $(A)$ de dimensiones $n \times n$ y una matriz de factores $(B)$ de $k \times n$ elementos estará en equilibrio cuando se satisfagan las condiciones de beneficio cero:

$$
\pi=\pi A+w B
$$

donde $\pi \mathrm{y} w$ son los vectores de precios de los bienes y de los factores, respectivamente; y en donde las actividades están normalizadas para producir una unidad de producto.

La ecuación (1) se puede representar como sigue:

$$
\pi(I-A)=w B
$$

y si suponemos que $(I-A)$ es no singular, se tiene:

$$
\pi=w B(I-A)-^{I}
$$

\section{Gráfica 1}

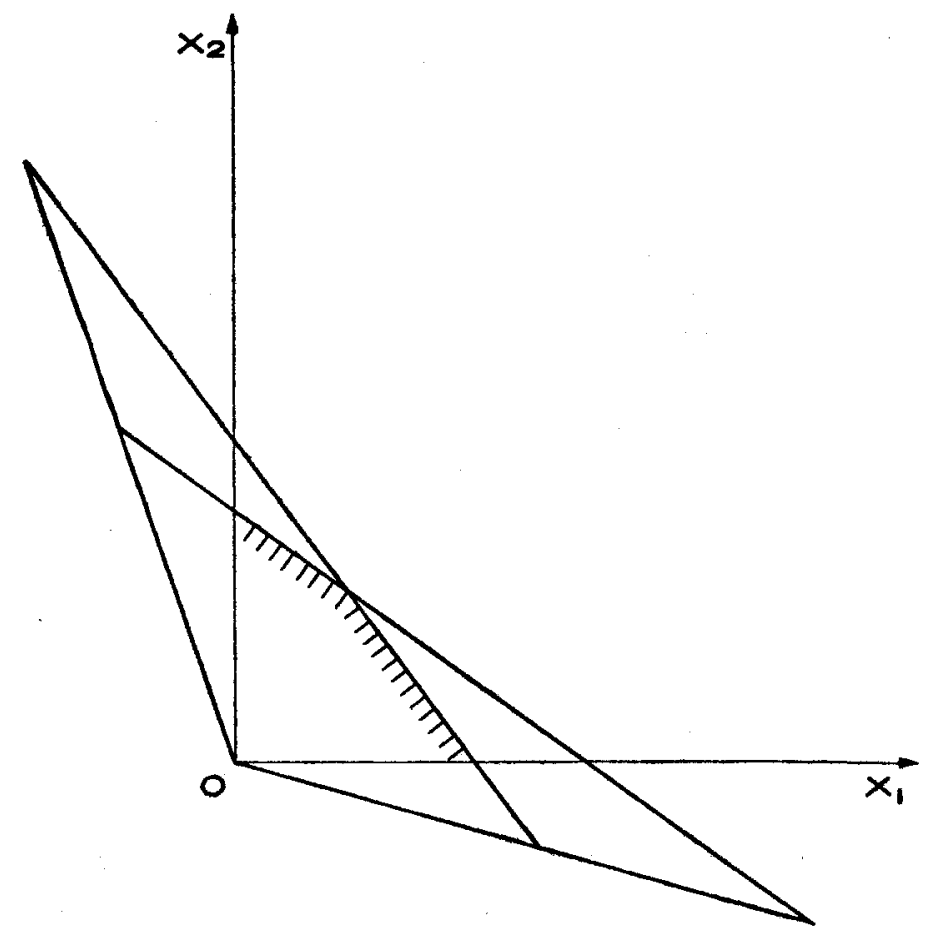


Como se muestra en la ecuación (3), los precios de los bienes están determinados por los precios de los factores cuando se conocen las actividades existentes. Se debe tener en cuenta que si existe un solo factor escaso, los precios relativos de los bienes son determinados por los coeficientes técnicos. Otra manera de expresar lo anterior, mediante un procedimiento gráfico - con una matriz de $2 \times 2$ - es que las fronteras posibles de producción no tendrán una inclinación única como se muestra en la gráfica 1.

Es claro que los precios de los bienes están determinados no sólo por los coeficientes técnicos; es necesario considerar el factor mercado en la estimación de los precios relativos para el mercado de bienes. En la siguiente sección se describe el algoritmo para la estimación de los precios de equilibrio para bienes y factores y en el cual se consideran estas interrelaciones.

\section{El Algoritmo}

El algoritmo que se utiliza en este trabajo no es original. Scarf (1973), utiliza un procedimiento similar cuando distingue entre bienes demandados (aquellos que se consideran en la función utilidad de los individuos) y bienes no demandados. Posteriormente Helpman (1973) utilizó también una técnica similar para encontrar soluciones de equilibrio general en un mundo con intercambio. De un modo simplificado, en nuestro trabajo se utiliza esta técnica al combinar la programación lineal y el algoritmo de Scarf: se considera sólo un país cuya economía se describe mediante una matriz de producción en la cual no se registra producción conjunta y se considera más de un factor escaso.

El algoritmo consiste en separar los dos mercados diferentes: el de factores y el de bienes. En otros términos, si existen $n$ bienes y $k$ factores, se podría resolver el problema de programación lineal para el mercado de bienes y utilizar el algoritmo de Scarf para el mercado de factores; esta separación permite la búsqueda de los precios de equilibrio de los factores en un sistema "simplex" de $k$ dimensiones. Sin la separación, la utilización del algoritmo de Scarf podría conducir a buscarlos en un sistema "simplex" de $n+k$ dimensiones. Veamos la explicación:

Supóngase que la economía produce $n$ bienes y que se dispone de una matriz de insumo-producto $(A)$ de $m \times n$ con producción no conjunta. Aquí se requiere que $m$ sea mayor o igual que $n$; suponemos que existe por lo menos una actividad para producir cada uno de los $n$ bienes pero que se permite la "substitución". Al mismo tiempo, se tienen $k$ factores de producción escasos cuya utilización en las actividades está dada por la matriz $B$ de $k \times m$.

Atenderemos primero el mercado de factores. Si sólo se dispone de tres factores, el sistema de solución podría representarse conforme a la gráfica 2.

En esta gráfica, los vértices de cada una de las subsoluciones repre- 


\section{Gráfica 2}

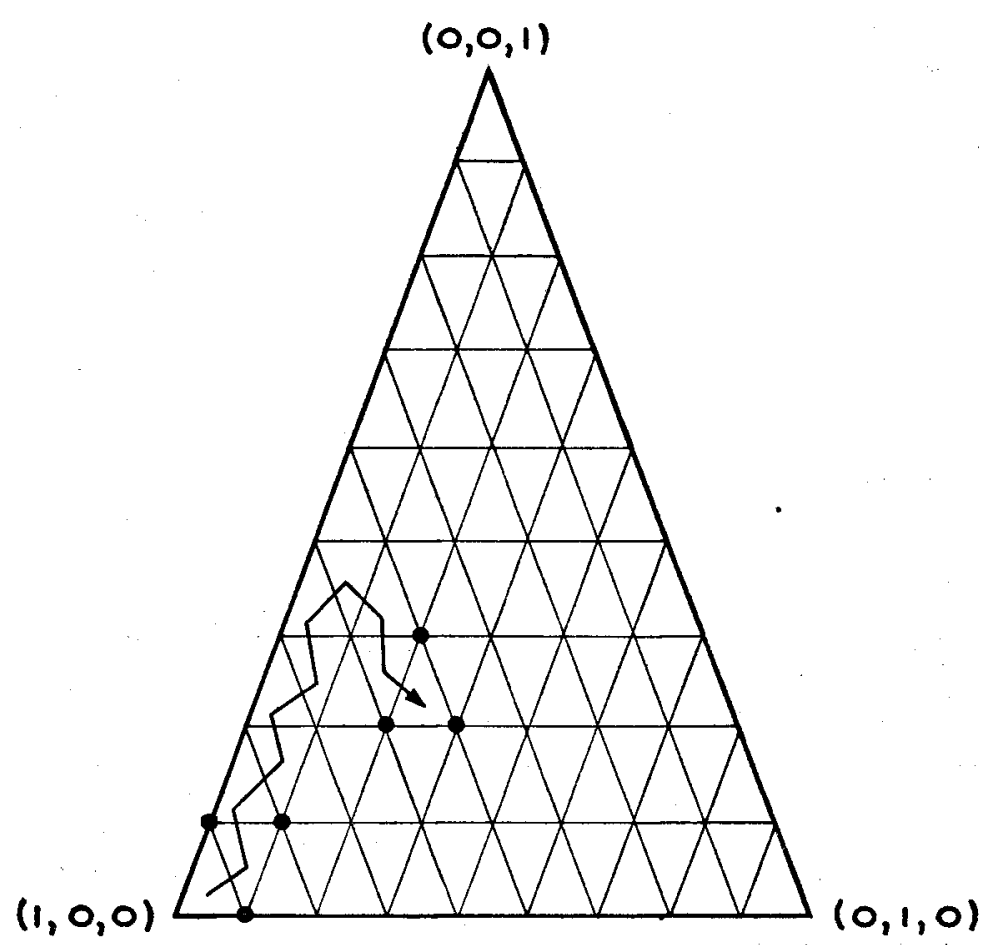

sentan conjuntos de precios de los factores: los precios de equilibrio serán aquellos representados por el vértice de una solución "completamente identificada"; o sea uno de tales vértices constituye una base factible para el problema adecuado de programación lineal, lo cual implica que el exceso de demandas de factores suman cero. ${ }^{2}$

La primera etapa del algoritmo consiste en escoger de manera arbitraria un conjunto de precios de los factores, digamos el punto $A$ en la gráfica 2. Dado este vector de precios de factores $\left(w^{o}\right)$, que está contenido en el modelo de solución y que en el caso general podría ser de dimensiones $k+1$, se pasa al mercado de bienes y se resuelve un problema de programación lineal mediante el cual se minimiza el costo de producción. Este problema tiene la siguiente forma:

$$
\begin{aligned}
\text { Encontrar Mín } w^{o} B x & \\
\text { sujeto a: } A x & \geqslant d \\
x & \geqslant o
\end{aligned}
$$

2 Para una descripción clara del proceso de "identificación" y el uso de teoremas de punto fijo véase Scarf (1960 y 1963). 
donde $x$ es un vector de niveles de actividad y $d$ es un vector arbitrario (lado derecho de la matriz) ${ }^{3}$

Mediante la solución de este problema se obtienen los precios sombra de los bienes $\pi^{o}$ así como los niveles de actividad $x^{*}$. A partir de este resultado se puede estimar la demanda final de los bienes o productos. Una vez estimada tal demanda final se puede resolver otro problema de programación lineal que reviste la siguiente forma:

\section{Problema 2}

Encontrar Mín $w^{o} B x$

$$
\text { sujeto a: } \begin{aligned}
A x & \geqslant d^{\prime} \\
x & \geqslant o
\end{aligned}
$$

donde ahora $d^{\prime}$ expresa el vector buscado de demandas finales.

La solución del problema 2 proporciona los mismos precios de equilibrio $\pi^{o}$ de los bienes y los niveles correctos de actividad $x^{* *}$. Con estos datos se puede estimar la demanda de factores buscada $\left(B X^{* *}\right)$. Estimado $B X^{* *}$ volvemos al mercado de factores para calcular el exceso de demanda: si los precios de los factores $w^{\circ}$ equilibran el mercado de factores, entonces el problema está resuelto. Si no, se utiliza el algoritmo de Scarf y se considera otro vector -en el "simplex" siguiendo digamos la trayectoria en la gráfica 2 - de precios de factores $w^{\mathbf{1}}$ y se resuelven de nuevo los problemas 1 y 2 ; y se sigue así el mismo procedimiento hasta obtener los precios de equilibrio de los factores. ${ }^{4}$ (El procedimiento del algoritmo se muestra en detalle en la sección siguiente).

Sin embargo, se debe garantizar que los precios $w$ y $\pi$ existen. A continuación se analiza este problema.

La existencia de $\pi$. Si se supone que la matriz $A$ es no-degenerativa, entonces tanto el problema 1 como el 2 tienen solución y el dual de precios es único: el vector $\pi$ es único. Esto es importante para los precios de los bienes.

La existencia de $w$. Para probar la existencia de los precios de los factores se utiliza el teorema de Debreu. ${ }^{6}$ Tal teorema establece que si $w$ y $\Phi(w)$ son respectivamente los precios de los factores y él conjunto de todos los vectores de excedentes de demanda de factores, y que si:

3 Se puede elegir $d$ de manera arbitraria dado que, conforme al teorema de no substitución, el resultado es independiente del lado derecho de la matriz.

4 Para cada $w^{i}$ se tiene un problema de programación lineal. Se podría pensar que esta técnica es muy laboriosa dado que en cada etapa se requiere la solución de dos problemas de programación lineal; esto no es del todo cierto, entre cada etapa sólo se cambiará la función objetivo del problema; en el peor de los casos - cuando se tienen más de $n$ actividades- sólo se tendrá que hacer un poco más que un paso pivotal. En la mayoría de los casos no se tendrá que hacer dado que los cambios en los precios de los factores son reducidos.

5 Véase Scarf (1973), p. 78.

6 Véase Debreu (1959), p. 82. 
i) para cada $w, \Phi(w)$ es un conjunto convexo, limitado, cerrado, $\mathrm{y}$ no-vacío;

ii) la proyección $w \rightarrow \Phi(w)$ es semicontinua superior;

iii) la proyección está acotada desde abajo en el sentido de que existe algún vector $b$ positivo con $p>-b$ para todo $p \in \Phi(w)$ y todo $w \in s$;

iv) si $p \in \Phi(w)$ entonces $w . p \leqslant 0$;

entonces existe $w^{*} \in s$ y $p^{*} \Phi\left(w^{*}\right)$ con $p^{*} \leqslant 0$.

En nuestra terminología $B x^{* *}{ }_{-} w$ representará el excedente de demanda de factores, en donde $w$ es el vector de oferta de factores productivos. Para verificar la existencia de $w$ se tiene que probar que $B x^{* * *}-w$ satisface las condiciones anteriores.

Los vectores de demanda de factores productivos, tales como $B x^{* *}$ se configuran con cada conjunto posible de demandas de bienes o productos. Dado que la demanda de factores son funciones lineales de las cantidades producidas, entonces el conjunto de vectores de demanda de factores, que es generado por un vector dado de precios de tales factores, es convexo dado que cualquier combinación convexa de dos conjuntos factibles es también un conjunto factible. Si las funciones de demanda están acotadas entonces este conjunto está también acotado. ${ }^{7}$ Por lo tanto, $\left(B x^{* *}-w\right)$ satisface la condición $(i)$. La proyección $w \rightarrow \Phi(w)$ es semicontinua superior. ${ }^{8}$ Dado el hecho de que las demandas de factores son no negativas, se puede construir el vector $\left(b_{1}+1, b_{z}+1, \ldots \ldots, b_{k}+1\right)$ que constituye el vector buscado $b$ de la condición (iii), donde $\left(b_{1}, \ldots b_{k}\right)$ es cualquier vector de demandas de factores productivos. Por último, se requiere verificar la ley de Walras, condición (iv). Por el teorema de dualidad de programación lineal se tiene:

$$
w^{*} B x^{* *}=\pi^{*} d^{\prime}
$$

Si todos los consumidores gastan todo lo que ganan:

$$
\pi^{*} d^{\prime}=w^{*} \mathrm{w}
$$

y, de (4) y (5):

$$
w^{*}\left[B x^{* *}-w\right]=0,
$$

que satisface la condición (iv) con estricta igualdad, y; por lo tanto $\pi \mathrm{y}$ $w$ existen.

7 Véase Helpman (1976), p. 551.

8 Véase Debreu (1959), p. 19, teorema 4. 


\section{UNA APLICACIÓN}

Se decidió presentar un ejemplo que permite la estimación analítica para mostrar la veracidad del algoritmo. Al mismo tiempo, se presenta este ejemplo para resolverlo manualmente (sin utilizar computadora) y mostrar las principales etapas del algoritmo. Resulta obvio que el algoritmo presentado antes puede utilizarse para problemas mayores: con $n$ bienes o productos y $k$ factores productivos.

En nuestro ejemplo se suponen:

a) dos bienes $\left(x_{1}\right.$ y $\left.x_{2}\right)$ y dos factores productivos ( $K$ y $\left.L\right)$;

b) dos consumidores con dos funciones utilidad;

c) oferta de factores productivos inelástica;

d) la matriz de producción permite la substitución pero no contiene producción conjunta.

Entonces se tiene:

a) por el lado de la producción:

$$
\begin{aligned}
& A=\left[\begin{array}{rrrrrr}
-1 & 0 & 7 & -2 & 4 & -1 \\
0 & -1 & -3 & 5 & -2 & 3
\end{array}\right] \begin{array}{l}
X_{1} \\
X_{2}
\end{array} \\
& B=\left[\begin{array}{rrrrrr}
0 & 0 & -3 & -1 & -4 & -3 \\
0 & 0 & -3 & -5 & -1 & -1
\end{array}\right] \begin{array}{l}
L \\
K
\end{array}
\end{aligned}
$$

\begin{tabular}{|c|c|c|c|c|}
\hline & Cons & Imidor 1 & & Consumidor 2 \\
\hline Función utilidad & $X_{1}^{1 / 3}$ & . $\quad X_{2}^{2 / 3}$ & Mín & . $\left[4 X_{1}, \frac{4}{3} X_{2}\right.$ \\
\hline \multirow{2}{*}{ Demanda de $X_{1}$} & 1 & $I^{1}$ & 1 & $I^{2}$ \\
\hline & $\overline{3}$ & $\overline{\pi_{1}}$ & $\overline{4}$ & $\frac{\pi_{1}}{4}+\frac{3 \pi_{2}}{4}$ \\
\hline \multirow{2}{*}{ Demanda de $X_{2}$} & 2 & $I^{1}$ & 3 & $I^{2}$ \\
\hline & $\overline{3}$ & $\overline{\pi_{2}}$ & $\overline{4}$ & $\frac{\pi_{1}}{4}+\frac{3 \pi_{2}}{4}$ \\
\hline $\begin{array}{l}\text { Dotación inicial } \\
\text { de trabajo }\end{array}$ & & 5 & & 10 \\
\hline $\begin{array}{l}\text { Dotación inicial } \\
\text { de capital }\end{array}$ & & 10 & & 5 \\
\hline
\end{tabular}

b) Por el lado de la demanda:

$I^{i}$ es el ingreso del consumidor $i$ y $\pi_{j}$ es el precio del bien $j$. 
Se requiere configurar el esquema "simplex" en el mercado de factores; con un tamaño de "cuadro" de tamaño 20, el esquema simplex se representa en la gráfica 3 que muestra todas las combinaciones de precios del capital y del trabajo que están contenidas en el cuadro.

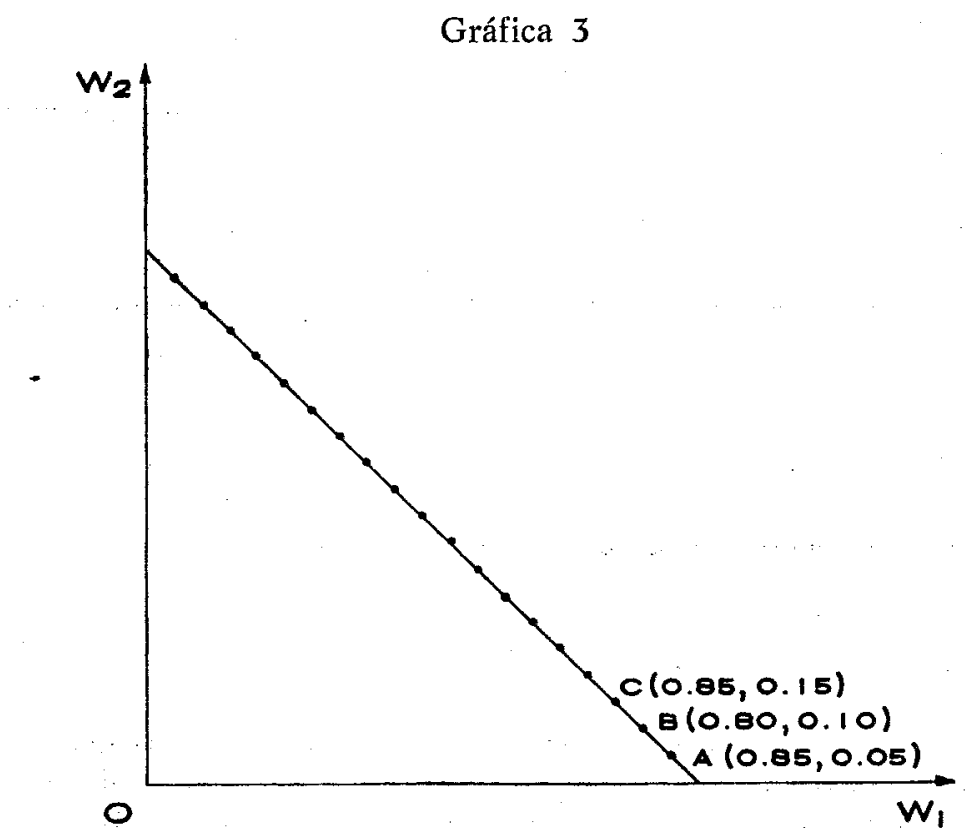

Se principia por la esquina inferior izquierda en el punto A $[\mathrm{W}=(0.95,0.05)]$; dados estos precios de los factores productivos se resuelve el siguiente problema de programación lineal:

Max. $-w B x$

$$
\text { Sujeto a: } \begin{aligned}
-A x & \leqslant-d \\
x & \geqslant 0
\end{aligned}
$$

La matriz inicial para el algoritmo "simplex" se representa mediante:

$$
\left[\begin{array}{ccccccccc}
1 & 0 & 0 & 3 & 1.2 & 3.85 & 2.95 & 10 & 0 \\
0 & 1 & 0 & -7 & 2 & -4 & 1 & -1 & -2 \\
0 & 0 & 1 & 3 & -5 & 2 & -3 & 1 & -1
\end{array}\right]^{0}
$$

- La octava columna es un vector $M$ que será utilizado en el cambio de "pivote" para evitar los valores negativos en el término del lado derecho; el término del lado derecho es equivalente al vector $d$ y ha sido elegido de manera arbitraria. 
Y después de cambiar de pivote cuatro veces se obtiene la siguiente matriz:

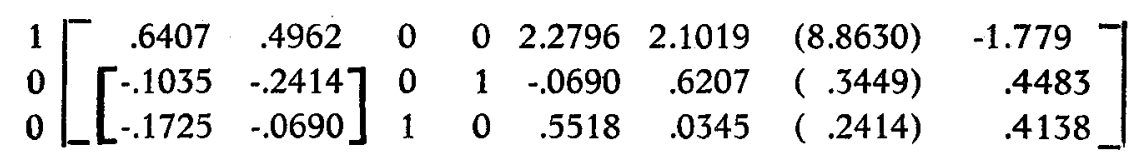

Se puede ignorar la octava columna (que aparece entre paréntesis) y los precios son:

$$
\pi=(0.6407,0.4962)
$$
dores:

Dados estos precios se pueden estimar los ingresos de los consumi-

$$
\begin{aligned}
& I^{1}=5.25 \\
& I^{2}=9.75
\end{aligned}
$$

y estimarse las demandas agregadas para $X_{1}$ y $X_{8}$ :

$$
\begin{aligned}
& f_{1}(\pi)=7.3106 \\
& f_{2}(\pi)=20.7732
\end{aligned}
$$

Ahora se utiliza este vector de demandas finales para calcular los niveles de actividad de equilibrio (el equivalente a la solución del problema 2 en la sección anterior). Las actividades consideradas son las dos primeras y se realizan a los niveles siguientes: 2.6944 y 5.7713 . Con este vector $x^{* *}$ se puede estimar la demanda deseada para los factores de producción, $B x^{* *}$ :

$$
\left[\begin{array}{ll}
3 & 1 \\
3 & 5
\end{array}\right]\left[\begin{array}{l}
2.6944 \\
5.7713
\end{array}\right]=\left[\begin{array}{l}
13.8545 \\
36.9397
\end{array}\right]
$$

Ahora es necesario volver al "simplex" correspondiente a los precios de los factores productivos; al hacer los cambios de pivote sobre el vector asociado con el punto $(0.95,0.05)$ (aquí asociamos el vector del nivel consistente de las demandas finales de factores productivos con cada vértice; el término del lado derecho contiene las dotaciones de factores):

$$
\left[\begin{array}{llll}
1 & 0 & 13.8545 & 15 \\
0 & 1 & 36.9397 & 15
\end{array}\right]
$$

Se resolverá el problema cuando el paso se inicia en el primer renglón. Dado que $w=(0.95,0.05)$ no equilibra el mercado de factores, se utiliza otro vector de precios de factores y se resuelve de nuevo todo el sis- 
tema (se pasa del punto $A$ al $B)$; ahora $w=(0.90,0.10)$ y la función objetivo cambia a

$$
\left(\begin{array}{llllll}
0 & 0 & 3 & 1.4 & 3.7 & 2.9
\end{array}\right)
$$

En este caso no se requiere cambio de pivote y se utilizan las mismas actividades; los precios de los bienes son $\pi=(0.6624,0.5449)$. Al estimar las demandas de factores productivos buscados, se encuentra que -como en el caso anterior- $w=(0.90,0.10)$ no equilibra el mercado de factores. Entonces elegimos otro conjunto - punto $C$-, y se efectúa el mismo proceso.

Después de la búsqueda en el "simplex" y utilizando 13 conjuntos diferentes de precios de factores - con sólo un cambio de pivote, en el último problema de programación lineal - se obtienen los siguientes resultados:

a) Soluciones del algoritmo (media de los dos vértices dél "subsimplex").

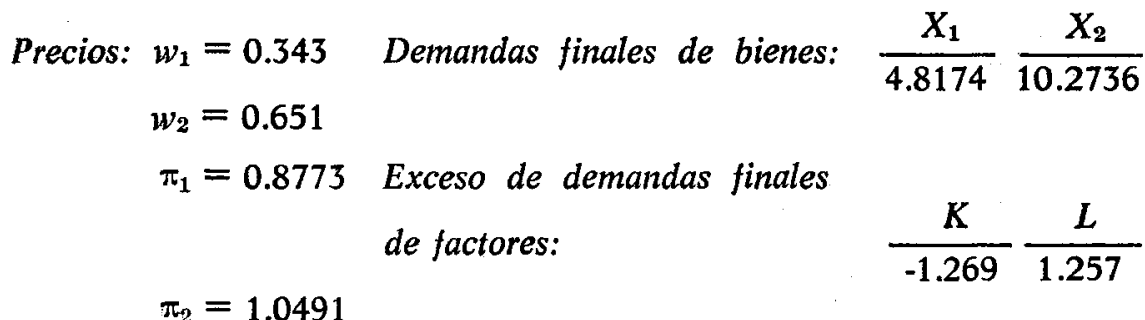

Niveles de actividad: $\frac{1}{1.4464} \frac{2}{1.3055} \frac{3}{0} \frac{4}{2.6953}$

b) Al resolver el mismo problema analíticamente se obtienen los siguientes resultados:

Precios: $w_{1}=0.3465$ Demanda final de bienes: $\quad \frac{X_{1}}{4.7233} \frac{X_{2}}{9.9992}$

$w_{2}=0.6535$

$\pi_{1}=0.8911$ Exceso de demandas finales

de factores:

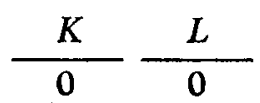

$\pi_{2}=1.0792$

Niveles de actividad: $\frac{1}{1.3890} \frac{2}{0.83331} \frac{3}{0} \frac{4}{3.3332}$

De manera obvia, la exactitud de la solución del algoritmo utilizado podría mejorarse al aumentar el tamaño de la escala (los excedentes de 
demanda de factores podrían tender a cero). Aun cuando nuestra escala es sólo de tamaño 20 , en nuestro caso se obtuvieron resultados bastante cercanos. El resultado notorio de este ejemplo es que en la solución final el sistema concluye con tres actividades, resultado que podría conducir a pensar en términos de alguna clase de proceso degenerativo pero, como se puede constatar, en este caso nuestro ejemplo es no degenerativo. Aun sin el proceso degenerativo, la economía está utilizando más actividades que bienes producidos. Este resultado conduce a la conjetura siguiente.

Conjetura ${ }^{10}$ Una economía que produce $n$ bienes, representada por una matriz de insumo-producto sin producción conjunta pero con posibilidades de substitución, y que utiliza $k$ factores $(k>1)$, puede incluir más de $n$ actividades; el límite superior es $n+k-1$.

\section{Conclusiones}

Este algoritmo utiliza matrices de insumo producto que no contienen producción conjunta y con más de un factor productivo escaso; dada la diferenciación hecha entre el mercado de factores y el de bienes productivos, esta técnica puede funcionar de manera eficiente con problemas de gran tamaño en términos de tiempo de computación.

2. Cuando se analiza una economía con más de un factor escaso, es superado el supuesto clásico de que los precios dependen sólo de la tecnología de producción; pero es necesario tener en cuenta el mercado de factores: los precios de los factores productivos determinan los precios de los bienes o artículos producidos.

3. Puede ser atenuado el supuesto de oferta inelástica de factores. Por ejemplo, se puede incluir el ocio - como un nuevo "artículo" o "producto"- al agregar una nueva actividad que produce montos positivos de ocio, con ceros en toda la matriz, excepto en el renglón de trabajo.

4. El algoritmo permite también el análisis de los efectos de distorsiones tales como impuestos y aranceles sobre el equilibrio general. Por ejemplo, en una economía abierta podríamos incluir aranceles en la matriz de factores al considerar otro "factor escaso" llamado aranceles (boletos) los cuales tendrían que ser comprados por el agente que utiliza dicha actividad a niveles positivos.

5. Una economía sin producción conjunta y más de un factor escaso y que permite la substitución, tendrá -en general- más actividades que bienes producidos; el límite superior será el número total de bienes más el número total de factores productivos menos uno. Entonces no parecería válida para el caso general la versión del teorema de substitución de Samuelson.

$10 \mathrm{El}$ Profr. Dietrich Fischer después de leer este trabajo en julio de 1977, verificó esta conjetura en su artículo "On the Number of Linear Economic Activities". 


\section{BIBLIOGRAFIA}

K. Arrow y F. Hahn (1971), General Competitive Analysis, San Francisco, Cal., Holden Day,

G. Debreu (1959), Theory of Value, New Haven, Conn., Yale University Press.

R. Dorfman, P. Samuelson y R. Solow (1958), Linear Programming and Economic Analysis, Nueva York, McGraw Hill.

E. Helpman (1976), "Solutions of General Equilibrium Problems for a Trading World", Econometrica, mayo de 1976.

H. Scarf (1969), "An Example of an Algorithm for Calculating General Equilibrium Prices", American Economic Review, septiembre de 1969.

H. Scarf (1973), The Computation of Economic Equilibria, New Haven. Conn., Yale University Press. 
FE DE ERRATAS

\section{del núm. 36 de Demografia y Economía, vol. XII,} núm. 3

1. p. 450 , segundo párrafo, últimas dos líneas; y p. 452, segundo párrafo, segunda línea:

dice: ... el factor mercado

debe decir: el mercado de factores

2. p. 453, gráfica 2: faltó ubicar la letra $A$ dentro del tercer triángulo pequeño (de izquierda a derecha) de la base del triángulo envolvente.

En la misma página, segundo párrafo, quinta línea:

dice: $k+1$

debe decir: $k \times 1$

$\mathrm{Y}$ en el último renglón de la nota 2 de pie de página:

dice (1960 y 1963)

debe decir: (1969 y 1973)

3. p. 454 , segundo párrafo, tercera y cuarta líneas:

dice: ...B $B X^{* *}$

debe decir: $B x^{* *}$

4. p. 455 , primer párrafo, última línea:

dice: ... $w^{*} \in s$ y $p^{*} \Phi\left(w^{*}\right) \ldots$

debe decir: ... $w^{*} \in s$ y $p^{*} \in \Phi\left(w^{*}\right)$

En la misma página, segundo párrafo, primera línea:

dice: ...B Bx**-w...

debe decir: $B x^{* *}-\omega$

En la fórmula 5):

dice: $\pi^{*} d^{*}=w^{*} w$

debe decir: $\pi^{*} d^{*}=w^{*} \omega$

$\mathrm{Y}$ en la siguiente fórmula:

dice: $w^{*}\left[B x^{* *}-w\right]=0$,

debe decir: $w^{*}\left[B x^{* *}-\omega\right]=0$

5. p. 457 , en la gráfica 3 : en el punto $B$ :

dice: $\mathrm{B}(0.80,0.10)$

debe decir: $\mathrm{B}(0.90,0.10)$

$\mathrm{y}$ en el punto $\mathrm{A}$ :

dice: $\mathrm{A}(0.85,0.05)$

debe decir: $A(0.95,0.05)$

En el segundo párrafo, primera línea:

dice: ... inferior izquierda ... .

debe decir: inferior derecha

$Y$ en el mismo párrafo, segunda línea:

dice: $\ldots[\mathrm{W}=(0.95,0.05)]$

debe decir: $[w=(0.95,0.05)$

6. p. 458 , en el párrafo penúltimo, tercer renglón:

dice: ....el vector del nivel...

debe decir: el vector de identificación 